\title{
Survivorship and Advocacy in Inflammatory Breast Cancer
}

\author{
Angela Alexander ${ }^{1,2,}$, , Terry Lynn Arnold 3 , Sandra Bishnoi ${ }^{4,5}$, Caroline Ballinger 6 , Simona F. Shaitelman? \\ Mark V. Schaverien, ${ }^{8}$ Lorenzo Cohen ${ }^{9}$, Mary Dev ${ }^{1}$, and Naoto T. Ueno ${ }^{1,2}$ \\ 1. Department of Breast Medical Oncology, The University of Texas MD Anderson Cancer Center, Houston TX \\ 2. Morgan Welch Inflammatory Breast Cancer Research Program and Clinic, The University of Texas MD Anderson Cancer Center, Houston TX, \\ 3. The IBC Network Foundation \\ 4. Rice 360 Program, Rice University, Houston TX \\ 5. Metastatic Breast Cancer Network \\ 6. The IBC Network UK \\ 7. Department of Radiation Oncology, The University of Texas MD Anderson Cancer Center, Houston TX \\ 8. Department of Plastic Surgery, The University of Texas MD Anderson Cancer Center, Houston TX \\ 9. Integrative Medicine Program, The University of Texas MD Anderson Cancer Center, Houston TX \\ $\bowtie$ Corresponding author: aalexand@mdanderson.org \\ (c) Ivyspring International Publisher. This is an open access article distributed under the terms of the Creative Commons Attribution (CC BY-NC) license \\ (https://creativecommons.org/licenses/by-nc/4.0/). See http://ivyspring.com/terms for full terms and conditions.
}

Received: 2017.06.01; Accepted: 2018.03.04; Published: 2018.04.06

\begin{abstract}
In February 2017, the Morgan Welch Inflammatory Breast Cancer (IBC) Research Program and Clinic hosted a scientific conference in Houston to commemorate the tenth anniversary of the opening of the first IBC-dedicated clinic in the world. Attendees included basic science researchers, clinicians who treat IBC, as well as patients and their caregivers. Several US-based and international IBC-focused nonprofit organizations were also represented. In this third paper from the conference, we report on the breakout session regarding survivorship and advocacy issues related to IBC, sharing an overview of the educational content presented and discussions regarding the future of IBC advocacy. Panelists focused on lymphedema research and clinical solutions, integrative medicine, and social work, with time provided for questions in small groups. IBC nonprofits that are leading advocacy efforts were introduced, and ways to become involved in these initiatives were discussed. Priorities for future advocacy and clinical care needs were also highlighted. In addition to summarizing these topics, we provide a suggested integrated IBC-specific plan of care that could be provided to the patient at the beginning of care and referred to throughout treatment and follow-up.
\end{abstract}

Key words: Inflammatory breast cancer, Advocacy, Survivorship needs, Patients, Lymphedema, Follow-up

\section{Introduction}

Inflammatory breast cancer (IBC) is a highly aggressive but uncommon presentation of breast cancer that has only recently been the subject of intense investigation as a distinct entity. In the 10 years since the formation of the Morgan Welch IBC Research Program and Clinic at MD Anderson Cancer Center, the multidisciplinary team has treated more than 600 patients with IBC. With the support of the state of Texas and New Mexico, a solid infrastructure has been developed to support laboratory-based and clinical research focused on improving the treatment and quality of life for patients with IBC. In addition, the clinic has valued the input of patient advocates and modeled ways to obtain mutual benefits by working together.

The program's 10th Anniversary Conference, held February 11-12, 2017, included a focus on survivorship, especially the experience of the patient during and after active treatment, and advocacy, which is defined broadly as activity that aims to influence decisions within political, economic, and social systems and healthcare institutions. 
The conference started with a presentation by $\mathrm{Dr}$ Sandra Bishnoi, an IBC patient advocate currently living with de novo metastatic disease. Addressing researchers, she passionately discussed the clinical relevance of research; she emphasized that saving patient lives should be the primary goal of research, and this can be aided by including patient advocates in research teams from the earliest planning stages through completion. Addressing patients and caregivers, she encouraged patients to be fully immersed in their care as equal partners with their oncologists, starting with reflection on personal priorities and including being well informed about their treatment options (clinical trials and standard of care).

Issues of survivorship and advocacy relevant to current patients, long-term survivors, and family members were the focus of a breakout session later in the day. A panel of faculty and staff from the areas of social work, radiation oncology, plastic surgery, and integrative medicine described resources available in their clinics. Key topics discussed in the session were lymphedema research and clinical solutions, integrative medicine and lifestyle changes during and after active treatment, the need for awareness of and access to clinical trials, In addition, the role of clinical trials in IBC treatments and a general overview of the types of trials, their purpose and characteristics of each were discussed, as well as challenges relating to travel and access to information about these trials. The final portion of the session focused on ways that patients and interested others can participate in advocacy relating to IBC, and clinical needs during survivorship, especially the need for a standardized IBC-specific follow-up plan. Important points and areas of need are addressed in the sections below.

\section{Lymphedema management and treatment}

The trimodal standard of care for IBC-neoadjuvant systemic therapy followed by mastectomy and post-mastectomy radiation-has been shown to lead to the best oncologic outcomes [1, 2]. However, such intense treatment has a cost in terms of long-term side effects and unique issues that IBC survivors face, few of which are well documented in the medical literature.

One major treatment-related sequela that often persists well beyond the end of active treatment is arm or truncal swelling, i.e., lymphedema. Post-treatment lymphedema arises in cancer patients who have surgery or nodal radiation since these local therapies damage the lymphatic system, thereby preventing proper drainage of lymph fluid to the circulatory system. As a result, lymph accumulates, usually in the ipsilateral arm in relation to the breast cancer, and if uncorrected, this fluid may harden. While there are no data specific to IBC on the incidence of lymphedema, our experience is that the majority of IBC survivors are affected, either immediately after treatment or significantly later in life (20-30 years later), indicating a lifetime continued risk for the development of this condition. Risk factors for lymphedema development include the extent of nodal surgery, axillary nodal radiation, and high BMI [3-5].

Despite lymphedema screening being included as a recommended component of standard of care in the National Comprehensive Cancer Network (NCCN) guidelines for breast cancer treatment since 2015, education/awareness about lymphedema has not become universally incorporated in routine IBC care in the community setting [6]. Patients with lymphedema assume that they feel that they must permanently endure this debilitating condition and may not seek solutions proactively. Given that lymphedema occurs in up to $40 \%$ of breast cancer survivors who were treated with local therapies, it would be prudent for all oncology team members to discuss this risk and potential strategies for early detection to ensure patients are receiving the highest quality of care [7].

The panelists' recommendations for prevention and early detection of lymphedema included exercising upper limbs to maintain/improve range of motion, maintaining a healthy weight, avoiding hand/arm injuries, and ensuring emergency care is sought at the earliest sign of potential cellulitis, a life-threatening infection that is more likely to become systemic in people with fewer lymph nodes than usual. In addition, patients with lymphedema should be encouraged to visit lymphedema-certified physical therapists to limit permanent reductions in functionality of their limbs.

Classic management of lymphedema has been limited to the wearing of compression bandages or garments, manual lymph drainage, and/or the use of lymphedema pumps that promote the drainage of lymph fluid into the circulatory system. A small study from Memorial Sloan Kettering Cancer Center identified acupuncture as an underutilized and noninvasive way to prevent progression of early lymphedema to a more serious state [8]. While these measures may halt the progression of lymphedema if initiated early, they are rarely a permanent solution, and such actions are time consuming and significantly decrease quality of life over the long term.

Over the past 10 years, surgeons at MD Anderson and other academic centers have been pioneering microsurgical procedures to correct acquired defects that lead to lymphedema [9]. These 
surgeries, once thought to be highly experimental and irreproducible, are now becoming standardized among the few centers that perform them. Dr Mark Schaverien of MD Anderson's Department of Plastic Surgery discussed some of these options. Examples of such surgical procedures include lymphovenous bypass and vascularized lymph node transfers, and in the most severe cases, liposuction of the fat that becomes deposited due to low lymphatic fluid drainage[10]. Liposuction is usually effective for arm volume reduction but requires the patient to wear compression garments continuously afterwards to prevent new fat buildup [11].

Modern lymphovenous bypass surgery involves near infrared lymphatic mapping using indocyanine green dye that is injected into the dermis in the arm to allow lymphatic vessel mapping and planning of bypass route. The blocked lymphatic vessels are then diverted to small adjacent veins, and intact lymphatic flow is verified using repeat dye tracking. In vascularized lymph node transfer, functional lymph nodes embedded in surrounding stroma are transplanted, usually from the groin or trunk region, to the axilla, which after recovery may open up an additional route for lymph flow into the blood. A recent review of the outcomes in such surgeries in a single center has been reported, and these results demonstrate that a $35-60 \%$ reduction in mean arm volume could be accomplished 1-12 months after surgery, and patients reported fewer symptoms such as arm heaviness or pain [12]. The magnitude of this change allowed patients to reduce the time spent in bandages from $76.8 \%$ to less than $10 \%$. As these procedures have become more effective, insurance companies are increasingly providing coverage for them, decreasing the financial barrier to surgery.

An approach for lymphedema prevention, called lymphatic microsurgical preventing healing approach (LYMPHA), has been proposed [13]. This strategy involves reverse axillary mapping and proactive anastomosing of arm lymphatics to adjacent veins at the time of mastectomy and axillary lymph node dissection. In one study of effectiveness of this approach, $87.5 \%$ of patients undergoing this procedure did not experience postoperative lymphedema, compared with $50 \%$ of patients who did not complete the procedure [14].

\section{Integrative medicine and lifestyle changes}

Integrative medicine, broadly covering the areas of diet, physical activity, stress management, and complementary therapies, is an important aspect of IBC survivorship. As described by Dr Lorenzo Cohen, the Integrative Medicine Center at MD Anderson and similar services at other large academic centers offer consults when patients have questions. Referrals from the patient's medical oncologists can be initiated at any point, whether during primary treatment (e.g. regarding the safe use of herbs and supplements during active treatment) or after active treatment (e.g., to discuss lifestyle changes to reduce risk of recurrence or deal with long-term side effects from treatment).

Often patients who are taking a large number of herbs and/or supplements do not realize the potential harmful effects these poorly regulated items may be having on their health. For example, in patients undergoing anthracycline-based chemotherapy or radiation, vitamins that act as antioxidants may blunt the therapeutic effects or potentiate the side effects of these therapies. In IBC, which is known to demonstrate intrinsic chemotherapy and radiation resistance, it is particularly important for oncologists to ask their patients about the use of vitamins, herbs, and supplements in a nonjudgmental manner and refer them to integrative medicine specialists if patients acknowledge taking such substances.

The topic of lifestyle changes for optimal health after treatment was also discussed, especially since high BMI is known to be a risk factor for IBC development, and being overweight is linked to worse outcomes in breast cancer [15, 16]. Unfortunately, some treatments may cause weight gain, and the side effects from therapy may make physical activity more difficult than before. Suggestions for increasing physical activity safely after treatment include small amounts of low-intensity exercise such as short walks, especially for people who previously led sedentary lifestyles.

An area of frequent concern is diet and whether certain foods should be avoided to minimize recurrence risk. Unfortunately, IBC-specific data regarding specific diet recommendations are lacking. However, Dr Cohen recommended as the basis for all cancer survivors eating a healthy plant-based diet, avoiding large swings in glucose levels (by eating a low-glycemic-load diet), and minimizing processed foods [17]. One common concern, especially among survivors of hormone-receptor-positive breast cancers, is soy, due to its known phytoestrogenic activity. Despite this theoretical concern, a recent meta-analysis has revealed that high dietary soy intake in its whole-food form is actually associated with lower risk of recurrence in both estrogen-receptor-positive and -negative breast cancers [18-20]. The many individual questions surrounding individual foods or vitamins asked in this session indicate a need for developing readily available accurate online resources on this topic. 
The role of stress management in cancer survivorship was another topic briefly discussed. In particular, incorporation of gentle forms of yoga after treatment was suggested as worthwhile and not requiring strong stamina to begin. The benefits of yoga include increasing flexibility and range of motion, and the deep breathing required benefits circulation and lymphatic flow, potentially helping with mild to moderate lymphedema, as well as other quality-of-life outcomes such as mood and sleep quality [21]. Adequate sleep (defined as at least 6.5 hours on average per night) was also mentioned as a benchmark for optimal post-cancer wellbeing, including decreasing the risk of a second breast cancer. Information on good sleep hygiene is available at www.sleepfoundation.org.

\section{Clinical trial awareness and resources enabling participation}

Self-advocacy and advocacy for others include learning about clinical trials that are available for IBC patients. There are several barriers to patients enrolling in such trials. One area is basic knowledge about how trials are designed, starting from the biological rationale to the different phases of trials and their goals and potential risks and benefits. A general overview of the types of trials and their characteristics was provided. The feedback obtained from attendees indicated that many patients, particularly those treated primarily in the community, lack basic awareness of these concepts, indicating that better outreach is needed by clinical investigators/research staff, perhaps in collaboration with research advocates who are more familiar with these topics. Even within large academic centers, trial navigation is far from seamless, and patients may miss opportunities if they don't specifically ask about these options for enhanced care.

Due to the low prevalence of IBC and historically poor outcomes, IBC patients have commonly been excluded from early-stage breast cancer studies and some metastatic cancer studies due to the concern of diluting a positive outcome in the larger population. Hence clinical trials that are focused on IBC tend to be investigator-initiated trials only open at a single academic center that houses an IBC clinic. Since discovering the availability of IBC-relevant clinical trials is a challenge for patients newly diagnosed with this rapidly progressing disease, a central patient-friendly resource is needed. The central U.S. government database of clinical trials, ClinicalTrials.gov, is thought to be overwhelming and difficult to navigate by patients, especially those with little background in cancer. A mobile application developed by an IBC nonprofit organization (The IBC
Network Foundation) fulfills this niche. In addition, a current list of available IBC-focused trials is provided in Table 1; however, other trials with agents that target proteins relevant to IBC pathogenesis certainly exist and may be reasonable options for certain patients. A complete list of these options is beyond the scope of this article. The key message from this talk was that patients should ask their oncologists about trial options early and frequently and not wait until they have exhausted all standard treatments and are seriously ill from their progressing cancer. IBC centers also may consider having dedicated staff to reach out to IBC patients regarding clinical trials that may benefit them, rather than relying solely upon the medical oncologist to discuss the trials.

Table 1: Currently enrolling IBC-focused clinical trials

\begin{tabular}{|c|c|}
\hline $\begin{array}{l}\text { ClinicalTrials.gov } \\
\text { identifier }\end{array}$ & Title \\
\hline \multicolumn{2}{|c|}{ Neoadjuvant Therapy } \\
\hline NCT02876107 & $\begin{array}{l}\text { A Phase II Study of Using } \\
\text { Panitumumab/Carboplatin/Paclitaxel (PaCT) Followed by } \\
\text { Anthracycline-Containing Regimen (AC) for New } \\
\text { Triple-Negative Inflammatory Breast Cancer (TN-IBC) }\end{array}$ \\
\hline NCT02623972 & $\begin{array}{l}\text { A Phase } 2 \text { Study of Eribulin Followed by AC as Preoperative } \\
\text { Therapy for HER2-negative Inflammatory Breast Cancer }\end{array}$ \\
\hline NCT02876302 & $\begin{array}{l}\text { Study of Ruxolitinib (INCB018424) With Preoperative } \\
\text { Chemotherapy For Triple Negative } \\
\text { Inflammatory Breast Cancer }\end{array}$ \\
\hline NCT01796197 & $\begin{array}{l}\text { Paclitaxel }+ \text { Trastuzumab }+ \text { Pertuzumab as Pre-Op for } \\
\text { Inflammatory BrCa }\end{array}$ \\
\hline NCT01525966 & $\begin{array}{l}\text { Carboplatin and Paclitaxel Albumin-Stabilized Nanoparticle } \\
\text { Formulation Before Surgery in Treating Patients With Locally } \\
\text { Advanced or Inflammatory Triple Negative Breast Cancer }\end{array}$ \\
\hline \multicolumn{2}{|l|}{ Adjuvant Therapy } \\
\hline NCT02971748 & $\begin{array}{l}\text { A Study of Anti-PD-1 (Pembrolizumab) + Hormonal Therapy } \\
\text { in HR-positive Localized IBC Patients With Non-pCR to } \\
\text { Neoadjuvant Chemotherapy }\end{array}$ \\
\hline \multicolumn{2}{|c|}{ Metastatic/Recurrent Disease } \\
\hline NCT02411656 & MK-3475 for Metastatic Inflammatory Breast Cancer (MIBC) \\
\hline NCT02658812 & $\begin{array}{l}\text { Talimogene Laherparepvec (T-VEC) for Breast Cancer Local } \\
\text { Recurrence }\end{array}$ \\
\hline NCT03101748 & $\begin{array}{l}\text { A Phase } 1 \text { b study of Neratinib, Pertuzumab and Trastuzumab } \\
\text { With Taxol (3HT) in Primary Metastatic and Locally Advanced } \\
\text { Breast Cancer, and Phase II study of } 3 \text { HT Followed by AC in } \\
\text { HER } 2+\text { Primary IBC, and Neratinib With Taxol (NT) Followe } \\
\text { by AC in HR+/HER2- Primary IBC }\end{array}$ \\
\hline NCT03202316 & $\begin{array}{l}\text { A Phase II Study of Triple Combination of Atezolizumab, } \\
\text { Cobimetinib + Eribulin (ACE) in Patients With } \\
\text { Recurrent/Metastatic Inflammatory Breast Cancer }\end{array}$ \\
\hline
\end{tabular}

Given the geographical clustering of IBC-focused clinical trials at large academic centers in the US, such as MD Anderson and Dana Farber Cancer Institute, one of the most common barriers to participation in clinical trials is the travel required for most patients. Our social work presenter, Ms Mary Dev, shared some resources available for travel, housing, and financial assistance for health care expenses through private foundations. Some of these require referral from the oncology team, so patients were urged to check into these resources and request help as early as possible to allow time for applications to determine the feasibility of travel for the right 
clinical trial. Academic centers often have social work counselors who provide supportive counseling and linkage with appropriate resources in the community.

\section{Opportunities for advocacy/education}

Patient involvement in research advocacy, as well as opportunities for legislative action and educating the public, was also discussed in the breakout session. These include efforts to fund/influence IBC research, and education regarding IBC standard of care and the need for specialized treatment for this rare and aggressive disease subset.

Research advocacy can involve a spectrum of tasks, including attending conferences (to learn and bring back research discoveries to peers), serving on grant review panels, and volunteering to be on academic committees related to clinical trial development. Patients without scientific backgrounds may initially be intimidated by these opportunities but were encouraged to participate. The demand for such advocates is predicted to increase quickly due to many funding sources asking explicitly for patient advocates to be named on grant applications, and the advocates are expected to be active participants in research teams to ensure that the research is clinically meaningful. Currently researchers not already affiliated with an institution with in-house advocates find it difficult to know where to begin to engage advocates and how to do so. The IBC Network Foundation seeks to fill this need to network patient advocates with researchers needing advocates; developing a database of interested patient advocates is a need. Resources for formal advocacy training were mentioned as opportunities for personal growth and networking for those interested.

A second issue related to research advocacy is the ongoing need for a unique medical code for IBC to more accurately define the prevalence of IBC and perform other clinical/epidemiological research. This issue unfortunately is not new, and is hindered by the complexity of IBC diagnosis, given that it is a clinical diagnosis with variable symptoms that can be highly subjective, as well as pathologically being indistinguishable from regular invasive carcinomas.

A huge area of need is health disparities with regard to IBC care. In recent studies of factors associated with use of trimodal care in IBC and survival outcomes, several socioeconomic and demographic factors were identified, including race, age, and insurance status [22]. Public health-related measures to address these are of urgent need to improve the use of the well-established IBC standard of care across the country, which would be predicted to increase survival in these patients.
Representatives of the 3 most established nonprofit IBC-focused organizations in the US and the newly formed IBC Network UK were present at the meeting. The IBC Network Foundation, the charity founded by Terry Arnold, a co-chair of the breakout session, focuses heavily on funding IBC-relevant research to directly help patients living with disease, but also provides patient support and educational resources. The Inflammatory Breast Cancer Research Foundation and Inflammatory Breast Cancer Foundation (also known as Erase IBC) focus on research and personal advocacy and on educating laypeople, respectively. Since all nonprofits, regardless of focus, need to raise funds to support their mission, survivors interested in raising money for these organizations were provided some suggestions for getting started and being persuasive in their communications about the cause.

The topic of education was also briefly mentioned, including the power of patients telling their story (accurately) and the power of social media in modern outreach. In recent years, mentions of IBC in lay media have increased significantly, yet many articles don't completely educate readers on the key differences between IBC and non-IBC, what to look for, and the importance of IBC specialty care. Survivors were encouraged to set up Google alerts for mentions of IBC so that they are aware of news articles discussing this disease. Being up to date on these articles, taking the time to comment on them, and sharing good articles on personal social media accounts were discussed as potential avenues for patients to contribute to expanding awareness of IBC without a need for travel or an extensive time commitment. In addition, following up on articles that could be improved can motivate follow-up articles that are more helpful.

A hard-copy 'advocacy toolkit' provided to the attendees included a list of resources for advocates to share with patients to facilitate clinical trial participation or travel to IBC specialty centers and included sample letters and postcards to use for lobbying or outreach. The need for advocates to specifically ask for IBC-focused research to be funded by federal, state, and nonprofit entities is pressing. In addition, a list of ways to become involved as a volunteer relating to advocacy was provided, and patients who desire these opportunities were encouraged to evaluate their skillsets and find a niche that utilizes these qualities. These materials may be obtained by contacting the corresponding author.

\section{Clinical needs regarding survivorship}

Finally, discussion among the panelists and participants highlighted the survivor's experience 
following the active treatment phase. During treatment, intense focus is placed on following the optimal treatment protocol (on a strict schedule) to survive this aggressive cancer; once active treatment is over, there is a need for support in going back to a more normal life without frequent appointments. After a year or more of very intense treatment, some patients have a sense of "freefalling" by not having a schedule for exams, testing, and bloodwork. Patients should be aware that the recovery time varies significantly, depending on differences in treatment, other health conditions, and the patient's life history, support system, and personality. For many patients, the period directly after active treatment can be very stressful, and patients who have recently finished treatment were encouraged to take care of their needs before jumping right into advocacy. Changes in personal circumstances, such as relationship challenges and work-related issues, are common problems post-treatment. Many women lose their jobs due to extended time away or must decrease their workload because of fatigue, pain, or mental challenges such as "chemo brain." The resulting loss of income can be very stressful, especially if there are additional healthcare expenses/debt due to cancer treatment.

One concern expressed was the lack of an IBC-specific follow-up plan and need for better coordination with healthcare providers who are not already a part of the IBC trimodal treatment team. Little standardization exists in follow-up plans for stage 3 IBC patients, which limits patient empowerment to deal with the questions that frequently arise post-treatment. Due to the higher rates of early recurrence in IBC, and the intensity of treatment involved in controlling the disease, often the follow-up/counseling needed is different from that for other early-stage cancers. Since many of our patients did not know about the additional services offered by integrative medicine and other consultative departments, we have incorporated these into a suggested integrated plan of care for IBC patients and survivors (Figure 1), to be provided by the primary oncology team to the patient at the beginning of their care and referred to throughout treatment and follow-up.

IBC survivorship research is an area of unmet need. Even in a dedicated IBC clinic, patients who have completed active treatment may not receive equal follow-up and education regarding ongoing

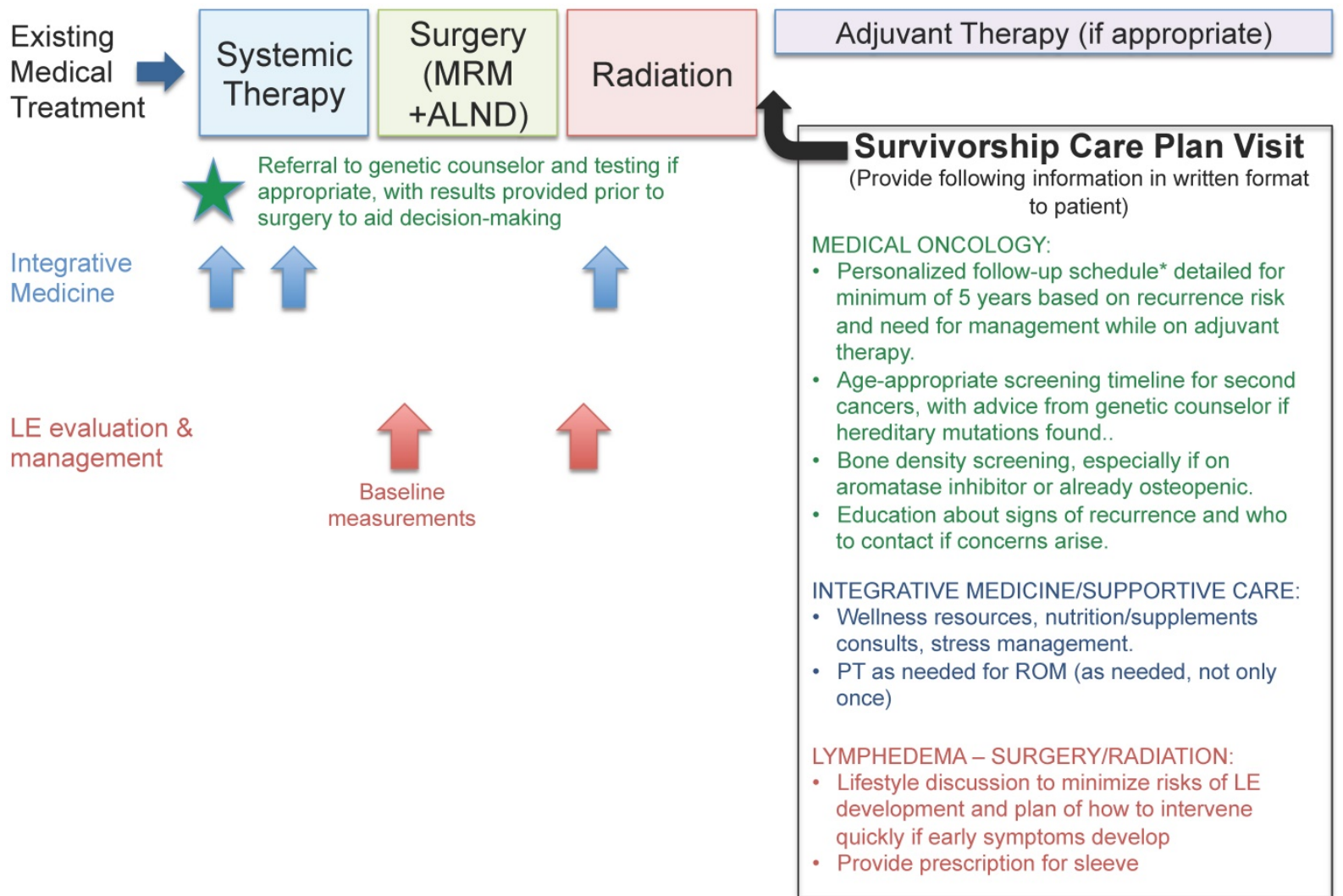

Figure 1. Integrative medical management for IBC patients and survivors. Schematic showing how to integrate both standard-of-care medical treatment and other supportive services, such as integrative medicine and lymphedema management, into IBC care. Suggestions for ideal components of a survivorship care plan include a standardized follow-up schedule of physician visits as well as referrals to other resources as needed. * Suggested follow-up schedule includes more visits during the early-recurrence period ( 2 years) (for example, every 3 months) and moves to a less frequent schedule if the patient is doing well (for example, for stage 3 patients, every 6 months during years 3-5 and then once a year if they remain disease-free). Stage 4 IBC management should be tailored to patients' disease features and response to therapies given. Abbreviations: MRM - modified radical mastectomy, ALND - axillary lymph node dissection, LE - lymphedema, ROM - range of motion. 
issues or potential sequelae from treatment. Survivors expressed a desire for more consistent care after radiation, rather than referral to a standard cancer survivorship clinic that may not be aware of specific needs of the IBC community. More research into the prevalence and severity of complications from treatment may inform these efforts and provide incentive to proactively offer solutions to patients. A comprehensive survivorship care plan published in the medical literature to include both medical needs (follow-up schedule and recommended monitoring component) and emotional needs would be of benefit to the IBC community at large. Given that the overall age distribution skews younger in IBC than in non-IBC, recommendations regarding issues such as fertility and the optimal timing of pregnancy are needed, particularly in the hormone-receptor-positive population, who are strongly encouraged to take long-term adjuvant endocrine therapy for recurrence risk reduction.

As leaders in IBC clinical care, we must consider how to help patients who have completed active treatment to return to their former lifestyle, realizing that a cancer diagnosis does cause some permanent changes in health and body image. From the survivor perspective, straightforward ways to help patients regain a sense of control include education to control or reduce long-term side effects and counseling regarding ways to reclaim their new lives post-treatment using forms of therapy, similar to what might be seen in PTSD. A growing number of helpful online support groups exist and may be worth mentioning to patients to complement their medical care at this crucial time. In addition, large centers could provide opportunities for peer mentoring, which can be empowering for both mentors and mentees, as a way to "pay it forward."

\section{Acknowledgements}

The authors wish to thank Sunita Patterson, senior scientific editor from MD Anderson Department of Scientific Publications for helpful comments during manuscript editing.

\section{Competing Interests}

The authors have declared that no competing interest exists.

\section{References}

1. Dawood S, Ueno NT, Valero V, et al. Identifying factors that impact survival among women with inflammatory breast cancer. Ann Oncol. 2012; 23: 870-5.

2. Dawood S, Merajver SD, Viens $P$, et al. International expert panel on inflammatory breast cancer: consensus statement for standardized diagnosis and treatment. Ann Oncol. 2011; 22: 515-23.

3. Tsai RJ, Dennis LK, Lynch CF, Snetselaar LG, Zamba GK, Scott-Conner C. The risk of developing arm lymphedema among breast cancer survivors: a meta-analysis of treatment factors. Ann Surg Oncol. 2009; 16: 1959-72.
4. Shaitelman SF, Chiang YJ, Griffin KD, et al. Radiation therapy targets and the risk of breast cancer-related lymphedema: a systematic review and network meta-analysis. Breast Cancer Res Treat. 2017; 162: 201-15.

5. Jammallo LS, Miller CL, Singer M, et al. Impact of body mass index and weight fluctuation on lymphedema risk in patients treated for breast cancer. Breast Cancer Res Treat. 2013; 142: 59-67.

6. NCCN guidelines for treatment: Breast Cancer. Version 2. April 6, 2017 ed: National Comprehensive Cancer Network (NCCN).

7. Norman SA, Localio AR, Potashnik SL, et al. Lymphedema in breast cancer survivors: incidence, degree, time course, treatment, and symptoms. J Clin Oncol. 2009; 27: 390-7.

8. Cassileth BR, Van Zee KJ, Yeung KS, et al. Acupuncture in the treatment of upper-limb lymphedema: results of a pilot study. Cancer. 2013; 119: 2455-61.

9. Allen RJ, Cheng $\mathrm{MH}$. Lymphedema surgery: Patient selection and an overview of surgical techniques. J Surg Oncol. 2016; 113: 923-31.

10. Smile TD, Tendulkar R, Schwarz G, et al. A Review of Treatment for Breast Cancer-Related Lymphedema: Paradigms for Clinical Practice. Am J Clin Oncol. 2018; 41: 178-80.

11. Schaverien MV, Munro KJ, Baker PA, Munnoch DA. Liposuction for chronic lymphoedema of the upper limb: 5 years of experience. J Plast Reconstr Aesthet Surg. 2012; 65: 935-42.

12. Gratzon A, Schultz J, Secrest K, Lee K, Feiner J, Klein RD. Clinical and Psychosocial Outcomes of Vascularized Lymph Node Transfer for the Treatment of Upper Extremity Lymphedema After Breast Cancer Therapy. Ann Surg Oncol. 2017; 24: 1475-81.

13. Casabona F, Bogliolo S, Ferrero S, Boccardo F, Campisi C. Axillary reverse mapping in breast cancer: a new microsurgical lymphatic-venous procedure in the prevention of arm lymphedema. Ann Surg Oncol. 2008; 15: 3318-9.

14. Feldman S, Bansil H, Ascherman J, et al. Single Institution Experience with Lymphatic Microsurgical Preventive Healing Approach (LYMPHA) for the Primary Prevention of Lymphedema. Ann Surg Oncol. 2015; 22: 3296-301.

15. Chang S, Alderfer JR, Asmar L, Buzdar AU. Inflammatory breast cancer survival: the role of obesity and menopausal status at diagnosis. Breast Cancer Res Treat. 2000; 64: 157-63.

16. Chang S, Buzdar AU, Hursting SD. Inflammatory breast cancer and body mass index. J Clin Oncol. 1998; 16: 3731-5.

17. Dieli-Conwright CM, Lee K, Kiwata JL. Reducing the Risk of Breast Cancer Recurrence: an Evaluation of the Effects and Mechanisms of Diet and Exercise. Curr Breast Cancer Rep. 2016; 8: 139-50.

18. Dong JY, Qin LQ. Soy isoflavones consumption and risk of breast cancer incidence or recurrence: a meta-analysis of prospective studies. Breast Cancer Res Treat. 2011; 125: 315-23.

19. Eakin A, Kelsberg G, Safranek S. Clinical Inquiry: Does high dietary soy intake affect a woman's risk of primary or recurrent breast cancer? J Fam Pract. 2015; 64: 660-2.

20. Fritz H, Seely D, Flower G, et al. Soy, red clover, and isoflavones and breast cancer: a systematic review. PLoS One. 2013; 8: e81968.

21. Greenlee H, DuPont-Reyes MJ, Balneaves LG, et al. Clinical practice guidelines on the evidence-based use of integrative therapies during and after breast cancer treatment. CA Cancer J Clin. 2017; 67: 194-232.

22. Denu RA, Hampton JM, Currey A, et al. Racial and Socioeconomic Disparities Are More Pronounced in Inflammatory Breast Cancer Than Other Breast Cancers. J Cancer Epidemiol. 2017; 2017: 7574946 